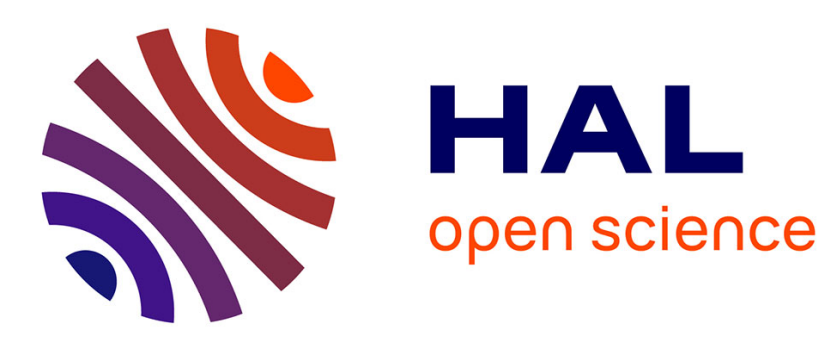

\title{
Cartozonage: de la carte au zonage du risque avalanche
} Richard Lambert

\section{To cite this version:}

Richard Lambert. Cartozonage: de la carte au zonage du risque avalanche. Neige et glace de montagne: Reconstitution, dynamiques, pratique, Collection EDYTEM - Cahiers de Géographie, nº, pp.233-237, 2009. halsde-00404062

\section{HAL Id: halsde-00404062 https://hal.science/halsde-00404062}

Submitted on 6 Apr 2011

HAL is a multi-disciplinary open access archive for the deposit and dissemination of scientific research documents, whether they are published or not. The documents may come from teaching and research institutions in France or abroad, or from public or private research centers.
L'archive ouverte pluridisciplinaire $\mathbf{H A L}$, est destinée au dépôt et à la diffusion de documents scientifiques de niveau recherche, publiés ou non, émanant des établissements d'enseignement et de recherche français ou étrangers, des laboratoires publics ou privés. 


\section{Collection EDYTEM}

Numéro 8 - Année 2009

Cahiers de

Géographie

\section{NEIGE et GLACE de MONTAGNE}

Reconstitution, dynamique, pratiques

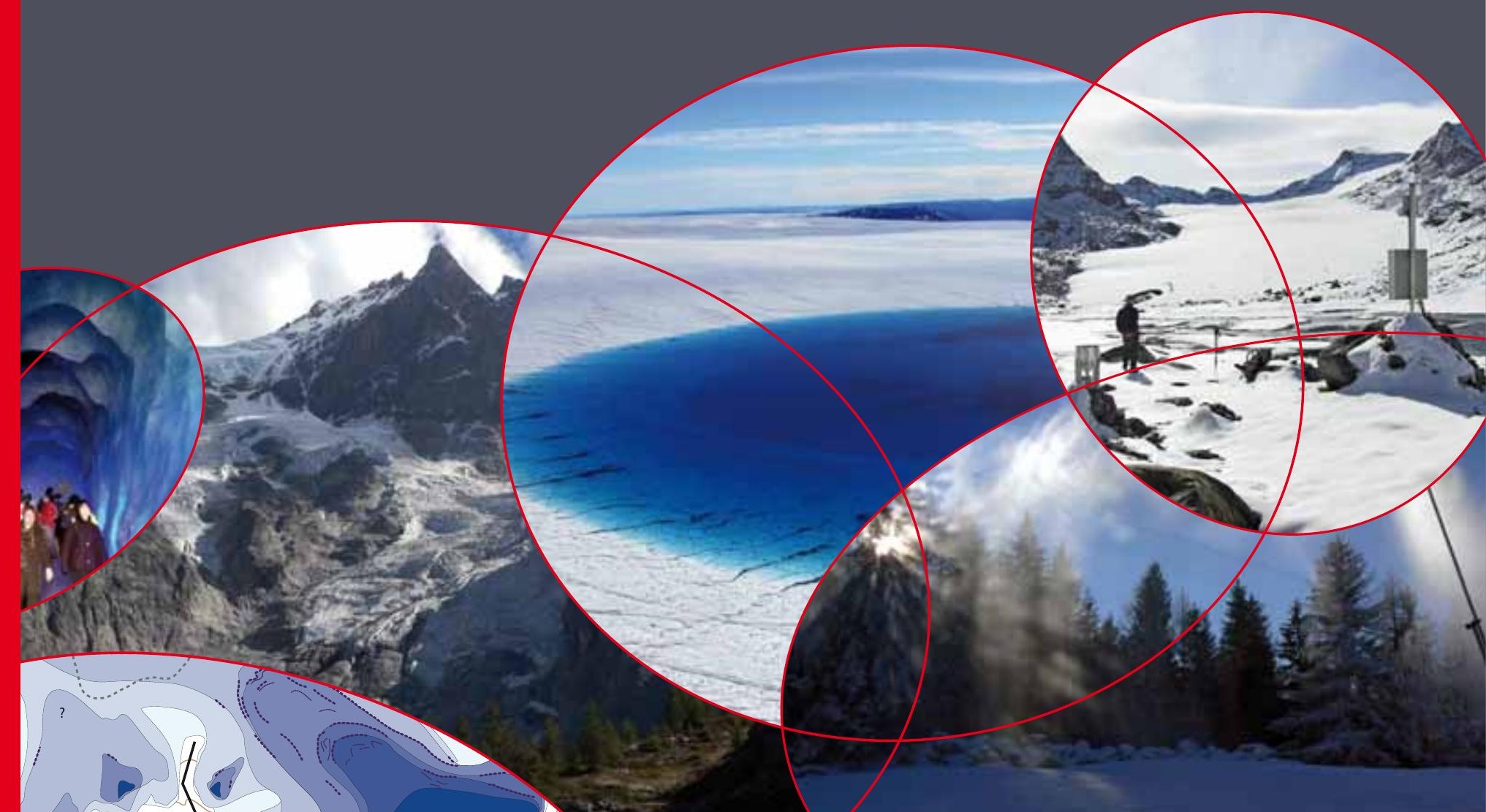





\title{
CARTOZONAGE
}

\section{DE LA CARTE AU ZONAGE DU RISQUE AVALANCHE}

\author{
CARTOZONAGE
}

FROM MAPPING TO ZONING OF THE AVALANCHE RISK

\author{
RICHARD LAMBERT
}

Laboratoire EDYTEM, Université de Savoie/CNRS, Campus scientifique, F 73376 Le Bourget-du-Lac cedex.

Contact : richard.lambert@univ-savoie.fr

\begin{abstract}
RÉSUMÉ
Cartographie et zonage représentent un enjeu actuel, essentiel pour la gestion spatiale du risque avalanche. Or ils sont étroitement liés dans leur recherche, leur évaluation et leur représentation graphique. La méthode concrète proposée ici repose sur 25 ans de pratique de l'expertise, et sur un constat : les documents Risques ont été souvent mis en défaut par des avalanches majeures mal prises en compte sur les cartes. La technique consiste à analyser un site donné, en additionnant les différents éléments repérés, puis de les présenter sur un seul document de synthèse final. Les expertises sont réalisées par une équipe pluridisciplinaire .

Sur un site urbanisé de montagne, exposé à de grandes avalanches (Vallée de Chamonix), la démarche a été expérimentée et a permis de proposer un zonage du risque en 4 zones.

L'intérêt de la méthode est de livrer aux responsables (élus, services de l'Etat) un produit facilement exploitable et mis en ceuvre rapidement : cartes d'avalanches, cartes d'aléas et zonage règlementaire sont acquis successivement et présentés ensemble, offrant ainsi une meilleure traçabilité des différentes analyses.
\end{abstract}

Mots CLÉS : AVALANCHES, RISQUES, CARTOGRAPHIE, ZONAGE, VALLÉE DE CHAMONIX.

\begin{abstract}
Cartography and zoning represent a vital challenge today for the spatial management of avalanche risk. They are indeed closely linked, in their research and evaluation as well as in their graphic representation. The concrete method proposed here is based on 25 years of expertise in avalanche assessment. The technique consists in analyzing a given site, adding up the different identified elements, and then presenting them in a single, final summary document. The principle is based on the successive superimposition of layers of information on an aerial photograph constituting the main background of the map. The expert assessments are carried out by a multidisciplinary team. This method was experimentally applied to an urbanized mountainous area exposed to several large avalanches (Chamonix valley).

The advantage of this method is that it provides the authorities with a finished product that they can immediately use. It also saves time, since avalanche maps, risk maps and regulatory zoning are acquired successively and presented together, each phase being justified by the previous phase, while at the same time applying a constant "double viewpoint", guaranteeing greater reliability.
\end{abstract}

KEYWORDS: AVALANCHES, RISKS, CARTOGRAPHY, ZONING, CHAMONIX VALLEY. 


\section{INTRODUCTION}

Cartographie et zonage représentent un enjeu très actuel, essentiel pour la gestion du risque avalanche, or ils sont étroitement liés : lorsque l'on réalise une cartographie d'avalanches, première étape obligée qui correspond au repérage spatial des phénomènes, la méthodologie d'investigation classique impose une recherche historique sur l'antériorité de survenance, une expertise sur le terrain avec analyse de la topographie et examen des traces géomorphologiques et dans la végétation. Ces éléments vont permettre de définir trajectoires, emprises latérale et longitudinale de l'avalanche, et d'évaluer intensité et fréquence. Ces deux paramètres sont déterminants pour établir la carte des aléas, étape principale pour présenter le zonage règlementaire.

\section{I - Constat : LeS limites de la Situation ACTUElle}

La méthode concrète proposée ici repose sur plus de 25 ans de pratique de l'expertise en avalanche et sur plusieurs constats :

- la dissociation marquée dans l'élaboration des cartes puis des zonages engendre des difficultés de report d'informations, voire des possibilités d'erreurs, en particulier par les différences d'échelles et de fonds de cartes utilisées pour les deux opérations ;

- même si l'expert collecte des informations essentielles pour le zonage, lors des investigations faites pour établir ou réviser une carte d'avalanche, celles-ci ne sont que partiellement reportées sur les documents finals, car le choix actuel, du moins en France, est de cantonner la cartographie d'avalanches à un simple « inventaire » des phénomènes constatés, où les notions plus « prospectives » d'intensité et de fréquence, ne sont pas considérées (Circulaire, 1970; CLPA);
- pourtant, lors de l'établissement d'un zonage pour un Plan de Prévention des Risques (PPR), le chargé d'étude doit connaître et considérer ces éléments plus prospectifs car ils sont l'architecture de base de la carte d'aléas. Aussi, il serait plus rationnel de mener toutes les investigations conjointement, avec une équipe pluridisciplinaire exerçant un constant double regard de contrôle (Lambert, 2004) ;

- les documents « Risques » établis selon le cahier des charges cadré par le Ministère de l'environnement, ont été parfois mis en défaut par des avalanches majeures mal prises en compte sur les cartes existantes dans les Alpes Françaises du Nord. Le cas des grandes avalanches de février 1999 est significatif : à Chamonix, 8 avalanches dépassant l'emprise inscrite sur les cartes d'avalanches ; avalanche mortelle en zone blanche de PER (Glass et al., 2000).

\section{II - UNE NOUVELLE MÉTHODE}

La technique consiste à procéder par étapes successives, pour aboutir à un seul document de synthèse : le principe repose sur la superposition de différentes couches d'informations, additionnées sur un fond de carte principal : une photographie aérienne type orthophotoplan, les informations liées à chaque étape étant portées sur un transparent. La démarche a été expérimentée sur plusieurs sites urbanisés de montagne, exposés à de grandes avalanches, c'est-à-dire dont la dénivellation dépasse 1200 mètres, avec des bassins d'alimentation potentiels larges. L'exemple présenté ici est celui de l'avalanche dite des Pélerins, encore appelée avalanche du tunnel du Mont Blanc ou des Glaciers, en vallée de Chamonix (figure 1).

\section{1 - La $1{ }^{\text {ère }}$ étape d'investigation : l'apport du spécialiste avalanche}

Par une expertise dite naturaliste réalisée sur l'ensemble du parcours de l'avalanche, complétée par l'examen depuis le versant opposé (ou par un survol en hélicoptère)

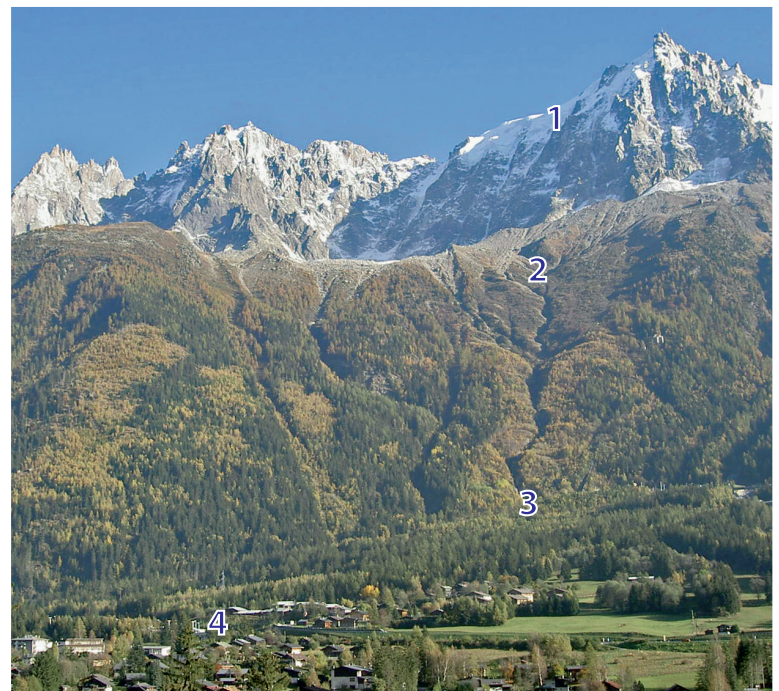

Figure 1 - La grande avalanche du Tunnel du Mont-Blanc (ou avalanche des Pélerins, ou des Glaciers), vue générale montrant :

1 - les zones de départ potentielles;

2 à 3 - la zone d'écoulement;

4 - la zone d'arrêt. 
sont déterminées zones de départ, trajectoires, emprises et extensions visibles ou retrouvées grâce à des dégâts précis dans la végétation ou à des traces géomorphologiques. L'analyse révèle en particulier différentes zones de départ potentielles, à différentes altitudes, et plusieurs zones d'écoulement possibles. Ces informations sont mémorisées conjointement sur une carte topographique au $1 / 10000^{\mathrm{e}}$ (ce qui permet un contrôle), puis reportées sur le fond orthophotoplan (figure 2).

Les apports de dendromorphologie étayent fréquemment ces approches et confirment les acquisitions techniques (Corona, 2007).

\section{2 - La $2^{\text {e }}$ étape : l'apport de l'historien}

Les recherches historiques sont souvent possibles dans nombre de vallées et massifs de montagne d'Europe où l'occupation humaine est fort ancienne.

« Là où une avalanche est venue, elle repassera » est une affirmation apparemment simpliste mais vérifiée sur plusieurs siècles de connaissance dans des sites très variés. Ces informations précieuses sont disponibles sous différentes formes : cahiers de raison, archives communales, puis plus tard, photographies, registres des Eaux et Forêts. Elles permettent de « remonter » à 2, 3 voire 4 ou 5 siècles.

Après vérifications d'usage (noms, localisation des lieux-dits, problèmes d'orthographe, etc.), ces recherches révèlent parfois des trajectoires inattendues ou des extensions exceptionnelles (figure 3).

Couramment, les éléments historiques obtenus confirment ce que l'expert avalanche a capté par la recherche de traces, dégâts, etc. Ces apports du passé sont donc croisés avec l'analyse du terrain : soit ils la valident parfaitement, soit ils la complètent. Il est important qu'ils viennent en phase 2, pour ne pas influencer la perception de l'expert avalanche confronté au terrain.

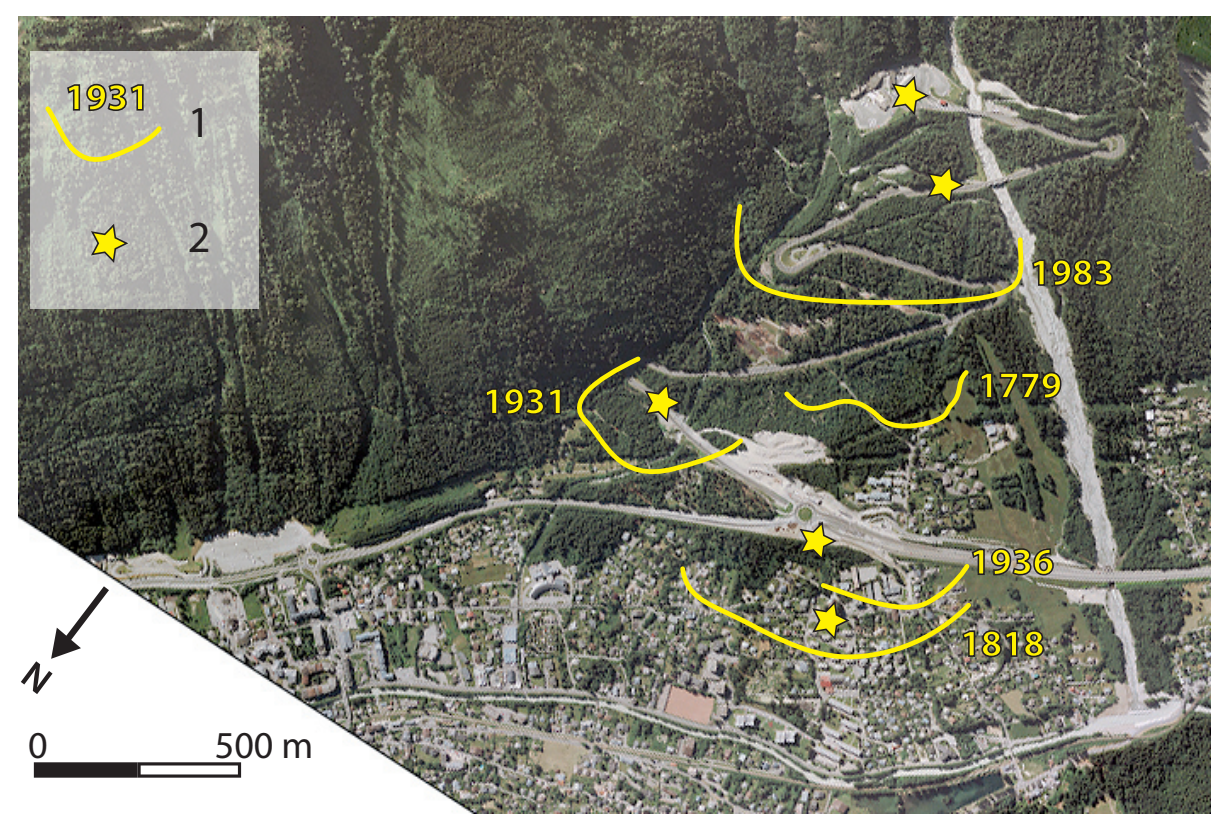

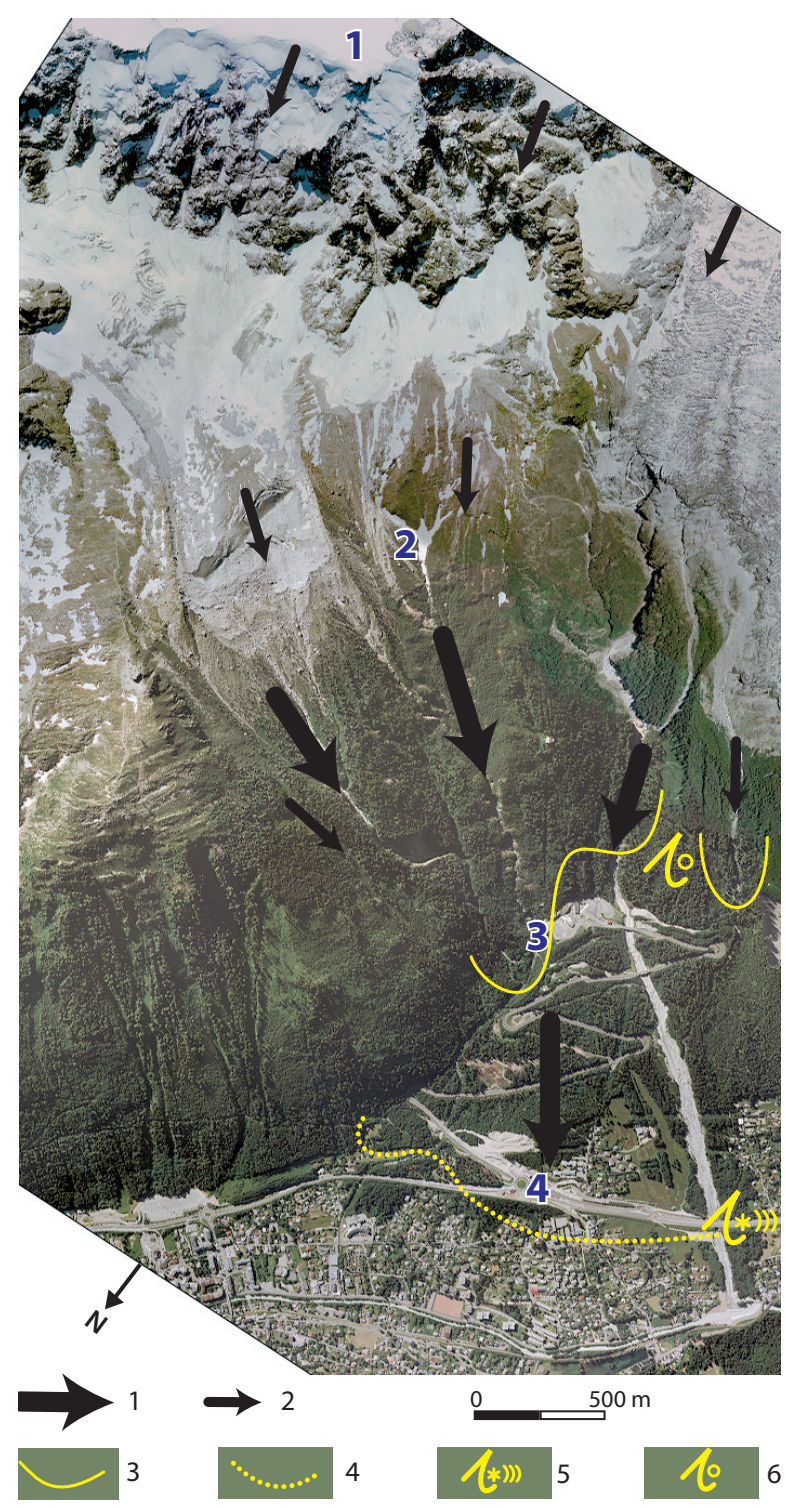

Figure 2 - La première couche d'informations : la carte d'avalanche préliminaire (les chiffres sur la photo ont la même légende que sur la figure 1) :

1 - trajectoire principale;

2 - départs ponctuels, flux secondaire,

3 - extension habituelle

4 - extension maximale probable;

5 - avalanche en aérosol avec effet de souffle,

6 - avalanche coulante, neige dense.

Fond $\mathrm{CIGN}$ - Paris - 2009 Extrait de données $B D$ ORTHO ${ }^{\circledR}$ Autorisation $n^{\circ}$ 80-9019

Figure 3 - La deuxième couche d'information : les contributions historiques:

1 - extensions maximales connues, avec année

\section{2 - dégâts.}

Fond $\mathbb{C I G N}$ - Paris - 2009 Extrait de donnée $B D$ ORTHO ${ }^{\circledR}$ Autorisation $n^{\circ} 80-9019$ 
Dans le cas présenté, une information historique fiable et dûment vérifiée, relatant un événement majeur en mars 1818, a parfaitement confirmé les analyses de terrain réalisées (figure 4).

Pour des sites à haute sensibilité en termes d'enjeux (forte occupation foncière...), il est possible d'avoir recours à une modélisation numérique pour affiner les distances d'arrêt des avalanches découvertes grâce aux recherches historiques. C'est une tendance récente.

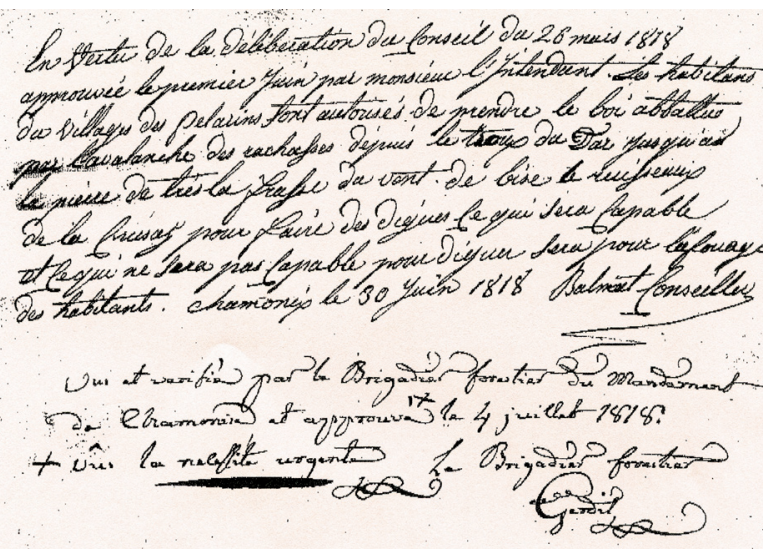

Figure 4 - Document de 1818 autorisant les habitants à prendre le bois abattu par l'avalanche des Rachasses (ancien nom de l'avalanche du Tunnel). Source: Association des Amis du Vieux Chamonix.

\section{3 - La $3^{\mathrm{e}}$ étape : la carte des aléas}

Cette carte doit distinguer les phénomènes courants des phénomènes dits exceptionnels, les emprises cartographiées variant en fonction des différentes intensités et des fréquences. Classiquement, on distingue l'aléa fort et l'aléa moyen.

L'aléa fort correspond à des intensités supérieures à $30 \mathrm{kPa}$ (kilo Pascals) et à des fréquences fortes ou modérées. Il est parfaitement justifié pour le secteur de l'entrée française du tunnel du Mont Blanc et la route d'accès située en contrebas, en regard de l'avalanche du 16 mai 1983 et des intensités constatées lors de cet événement majeur (figure 5).

L'aléa moyen correspond à des intensités comprises entre 1 et $30 \mathrm{kPa}$, associées généralement à des probabilités de retour modérées. Cette zone de «transition » où les intensités des phénomènes potentiels vont en diminuant, comprend le quartier haut du village des Pélerins, dont l'auberge de jeunesse.

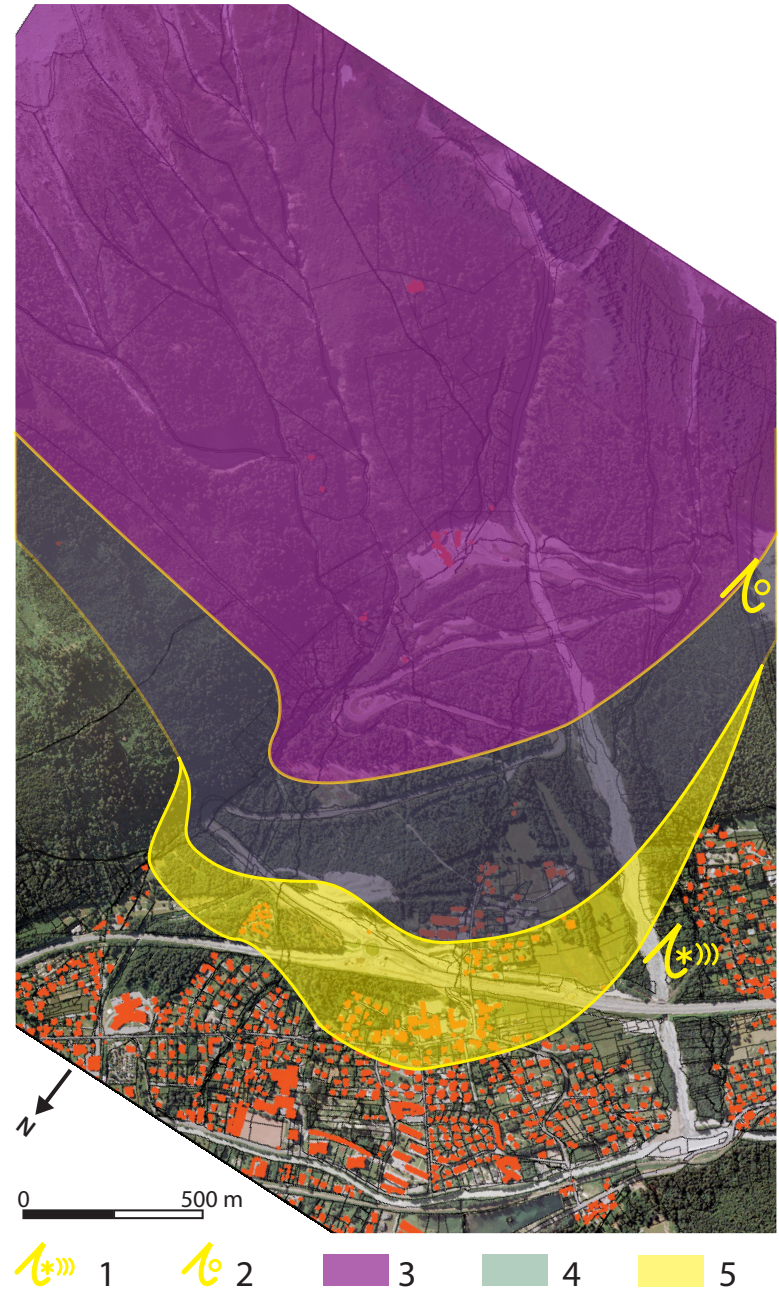

Figure 5 - La troisième couche d'information : la carte des aléas :

1 - avalanche en aérosol plus effet de souffle;

2 - avalanche coulante, neige dense;

3 - aléa fort (forte intensité et fréquence élevée) ;

4 - aléa modéré (intensité moyenne);

5 - aléa "exceptionnel» (intensité forte ou moyenne et fréquence faible), dégâts liés à une probabilité de retour pluricentennale.

Fond $\mathbb{C} I G N$ - Paris - 2009 Extrait de données BD ORTHO ${ }^{\circledR}$ Autorisation n $n^{\circ}$ 80-9019

L'aléa exceptionnel correspond à des phénomènes d'intensité forte, voire moyenne, mais surtout à une probabilité de retour rare à très rare (dépassant le siècle). Les informations acquises par les études historiques, en particulier les événements de 1818 et 1936, autorisent le tracé retenu qui englobe par exemple le quartier du groupe scolaire.

\section{III - LES RÉSULTATS CONCRETS, LA $4^{\mathrm{e}}$ ÉTAPE : LE ZONAGE RÈGLEMENTAIRE}

Ce document (figure 6) fait la synthèse de tous les apports en superposant toutes les couches de connaissance acquises.

Il est proposé ici un zonage du risque avec 4 couleurs, pour 4 zones (Lambert, 2004) :
- blanc : zone présumée sans risques, constructible ;

- rouge : zone inconstructible car concernée par des risques forts (découlant de l'aléa fort avec une probabilité d'occurrence moyenne à forte) ; 
- rose : zone concernée par des risques modérés (découlant de l'aléa moyen). C'est une zone intermédiaire entre rouge et blanc, de transition progressive : il a été jugé utile d'être plus proche de la réalité de l'écoulement d'une avalanche et de la diminution, en principe progressive, des intensités en allant vers l'aval. D'où la définition de 2 voire 3 zones « roses » d'intensité dégressive en s'éloignant du rouge. Cette zone est constructible sous certaines conditions, en particulier des prescriptions architecturales adaptées aux différents types d'avalanches susceptibles de s'y produire. Elle correspond à la zone bleue des actuels PPR français.

- jaune : zone concernée par les phénomènes extrêmes, pluricentennaux (200 à 400 ans) ; elle correspond à l'Aléa Maximal Vraisemblable (AMV) des PPR avalanches en France. Sa présence sur le zonage règlementaire est très importante : en effet, il paraît dangereux de fixer seulement un Aléa de Référence Centennal (ARC), classiquement retenu pour les risques naturels en France, car il est trop limitatif en regard des risques dits climatiques et ce choix entraîne de fait l'occultation des risques majeurs.

Souvent acquise grâce aux informations historiques, confirmée par une modélisation et/ou à « dire d'expert ", cette zone jaune fera apparaître des emprises latérales et surtout longitudinales correspondant à des avalanches de fréquence rare. Les

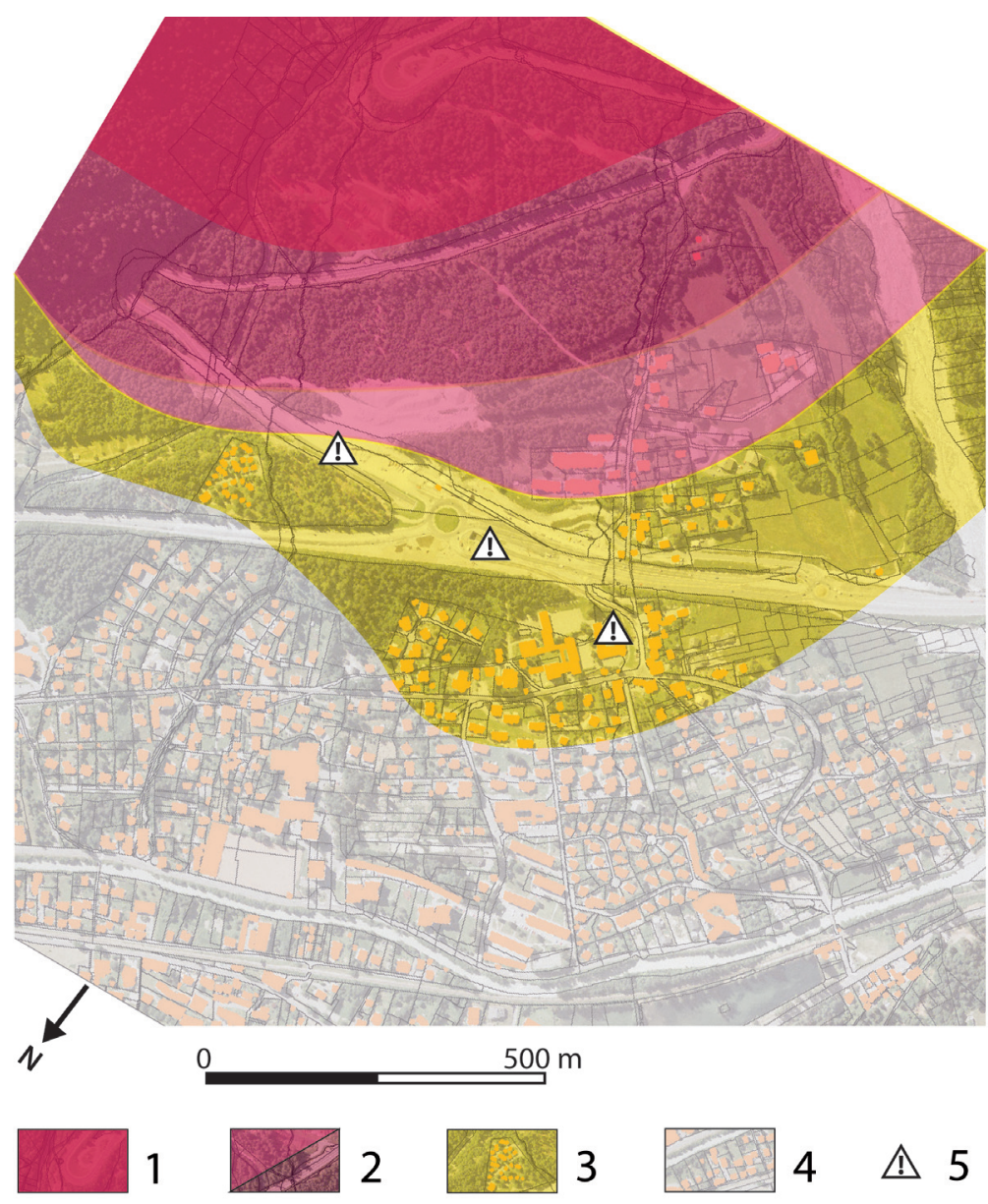

Figure 6 - La quatrième couche d'information: le zonage réglementaire. 1 - zone rouge, risque fort. Inconstructible;

2 - zone intermédiaire, risque modéré avec transition progressive de l'intensité. Constructible sous conditions (prescriptions particulières, contraintes architecturales, etc) ;

3 - zone jaune, phénomènes exceptionnels ou extrêmes. Constructible sous conditions ;

4 - zone blanche, présumée sans risques ;

5 -forts enjeux et/ou vulnérabilité préoccupante (route internationale, école, etc).

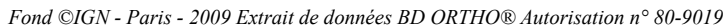

différences de distance entre les limites des zones rouges et jaunes peuvent être considérables (plus de 300 mètres dans l'exemple choisi ici). Exceptionnellement, la zone jaune est utilisée pour révéler une trajectoire d'écoulement rarissime. Enfin, elle matérialise des problèmes qui pourront apparaître suite à la disparition ou l'endommagement d'une forêt de protection en amont, ou si d'autres facteurs viennent à modifier la dangerosité d'un site de façon significative (formation de glaciers suspendus par exemple).

Les variations climatiques ne peuvent être mises en avant pour chercher à minimiser les risques d'avalanches au XXI siècle, par rapport aux événements connus de la fin du Petit Age Glaciaire : rappelons que « l'hiver du siècle » (1999) est intervenu durant la décennie la plus chaude du $\mathrm{XX}^{\mathrm{e}}$ siècle !

Cette zone jaune, qui n'apparaît pas forcément pour chaque avalanche analysée, est un élément de sécurité supplémentaire. Zones rouges et zones intermédiaires concernent en priorité le foncier, donc la protection des biens. La zone jaune, elle, vise exclusivement à améliorer la sécurité des personnes. Elle doit rester constructible.

C'est d'ailleurs selon cette philosophie qu'elle fut définie dans le guide méthodologique des PPR avalanches, comme l'AMV par opposition à l'ARC (Ministère, 2004).

Si des conditions nivométéorologiques particulièrement préoccupantes susceptibles de produire un phénomène d'une emprise exceptionnelle sont repérées, les usagers concernés spatialement sont prévenus et, selon le cas, évacués ou confinés. C'est là tout l'enjeu des Plans Communaux de Sauvegarde qui, pour être efficaces, doivent préalablement reposer sur un zonage le plus fiable possible (Loi 2004-811). 


\section{Conclusion}

L'utilité de cette nouvelle méthode est de fournir un produit fini, rapidement exploitable pour situer et gérer le risque. La chaîne d'élaboration du document final est visible, la traçabilité de la connaissance acquise est plus transparente. Sur un même document, le phénomène général depuis sa zone de départ et le cas particulier en zone d'arrêt sont présents et représentés.

Le danger de la situation française actuelle en matière de risque est la dichotomie dans les obligations et implicitement les responsabilités :

- l'Etat affiche le risque ;

- le Maire gère le risque.
Ainsi le second se voit imposer par le premier une situation souvent très délicate à gérer. Or l'Etat n'a pas, pour le moment, de réelles obligations pour conseiller les élus et les aider à la décision. Les usagers sont aussi directement concernés en terme de sécurité. La présentation officielle des documents risques induit la notion de risque accepté. Il est plus facile pour un riverain exposé à un danger de comprendre et d'accepter les «règles du jeu» s'il en est mieux informé. A terme, la meilleure fiabilité du document, au moins garantie par le «double regard» exercé lors de son élaboration, peut optimiser l'efficacité des relations entre Etat, élus et usagers.

\section{BibLIOGRAPHIE}

CIRCUlaire $\mathrm{n}^{\circ} 3807 \mathrm{PN} / \mathrm{SI}$ du 16 novembre 1970, ministère de l'Agriculture, portant sur l'établissement de la carte inventaire des avalanches.

Corona C., 2007. Evolution biostasique du paysage, géodynamique nivéo-glaciaire et fluctuations climatiques récentes dans la haute vallée de la Romanche - Alpes du Nord. Thèse de doctorat, Institut de géographie alpine, Université Joseph Fourier, Grenoble.

Glass B., Huet P., Rat M., Tordjamm R., 2000. Inspection Générale de l'Environnement. Retour d'expérience sur l'avalanche du 9 février 1999 à Montroc, commune de Chamonix. Ministère de l'Aménagement du Territoire et de l'Environnement, $69 \mathrm{p}$.
LAMBert R., 2004. La gestion spatiale du risque avalanche, une question d'actualité. In Proceedings of the 10th symposium Interpraevent, Riva del Garda, Italia, 25-27, 163-171.

Loi de modernisation de la SÉcurité Civile. $\mathrm{n}^{\circ}$ 2004-811 du 13 août 2004. Article 13. http://www. legifrance.gouv.fr

Ministère de L'AmÉnAgEment du Territoire et de l'Environnement et Ministère de l'Equipement, des Transports et du Logement, 1997. Plans de Prévention des Risques naturels prévisibles (PPR), Guide général. La Documentation Française, 76 p.

Ministère De L'Environnement. Guide méthodologique et plans de prévention des risques d'avalanches : http://www. prim.net/professionnel/documentation/guide_avalanche/ page 01. html 



\section{NEIGE et GLACE de MONTAGNE \\ Reconstitution, dynamique, pratiques}

\section{Sommaire}

Editorial

Introduction

\section{1 - Reconstitution}

Coutterand S. et al. - Le lobe glaciaire lyonnais au maximum würmien : glacier du Rhône ou/et glaciers savoyards ?

Ravanel L. et al. - Désenglacement du haut bassin versant du Vorz (massif de Belledonne, Isère), au Tardiglaciaire et à l'Holocène.

Rey P.-J. - Sociétés et fluctuations du climat dans les Alpes nord-occidentales au Néolithique moyen.

Le Roy et al. - Étude des fluctuations glaciaires du Petit Âge de Glace dans le Massif des Écrins : apports de la lichénometrie.

Kirkbride M.P. - Datation des moraines holocènes d'Islande par tephrochronologie : un état de l'art.

Le Roy et al. - La dendroglaciologie, ou l'apport de l'étude des cernes d'arbres pour la reconstitution des fluctuations glaciaires holocènes.

Rabatel A. - Évolution glaciaire dans les andes subtropicales chiliennes entre 1955 et 2007 : conséquences pour la ressource en eau.

Le Roy et al. - Un inventaire des aérophotographies du massif du Mont Blanc.

\section{2 - Dynamique}

Ravanel L. - Évolution géomorphologique de la haute montagne alpine dans le contexte actuel de réchauffement climatique.

Gruber S. - Le permafrost de haute montagne.

Deline P. et al. - L'Aiguille du Midi (massif du Mont Blanc) : un site remarquable pour l'étude du permafrost des parois d'altitude.

Saulnier G.-M. et al. - Un éléphant volant est-il un oiseau ? Perspectives pour l'observation hydrométéorologique des milieux de montagne.

Jobard S. - L'instrumentation du glacier du Baounet : quels apports pour la traçabilité des mesures environnementales ?

Moreau L. - L'exploration du cryokarst glaciaire et son intérêt scientifique pour l'étude du drainage des eaux de fonte.

Mazué R. et al. - Suivi de l'évolution de la couverture detritique d'un glacier noir par photo-comparaison : le glacier d'Estelette.

\section{3 - Pratiques}

Paccard P. - Réchauffement climatique et ressource neige en domaines skiables.

Gauchon C. - Les hivers sans neige et l'économie des sports d'hiver : un phénomène récurrent, une problématique toujours renouvelée.

Laslaz L. - L'exclusion des glaciers des zones centrales des Parcs nationaux de la Vanoise et des Écrins et leur équipement pour le ski d'été.

Cayla N. - Les sentiers d'interprétation glaciaire : des outils de valorisation différenciée des glaciers et de leur territoire.

Lambert R. - Cartozonage : de la carte au zonage du risque avalanche.

Moulin A. et al. - L'incertitude liée aux avalanches dans les Alpes du Nord : identifications et implications pour la gestion.

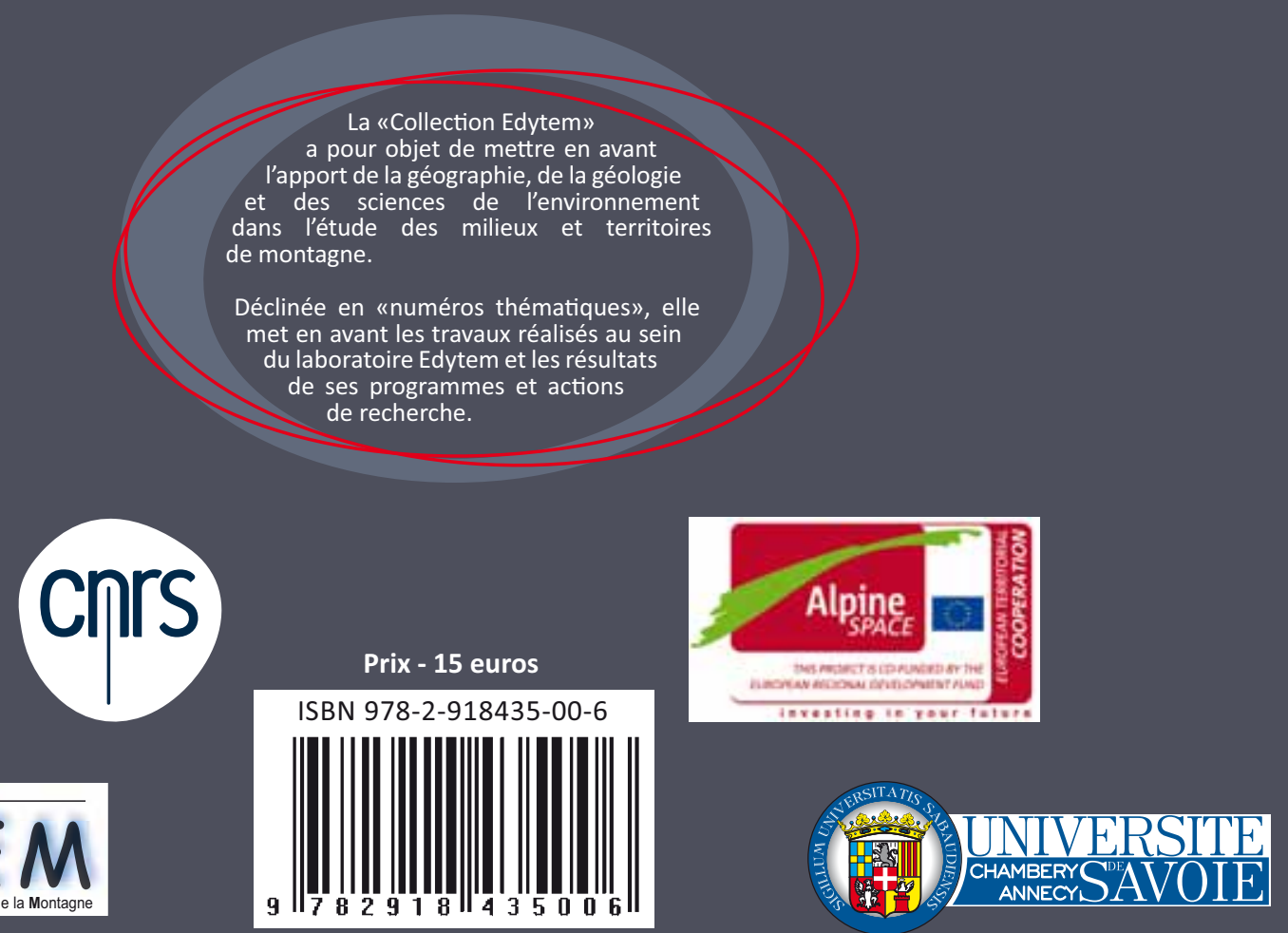

